\title{
Clinical characteristics of pediatric cases of SARS-CoV- 2 infection in Hunan, China: A retrospective, multi-center case series
}

Lei Wu

Changsha Central Hospital of South China University

Xiao-Fo Zhang

Changsha Central Hospital of South China University

Jia Li

Changsha Central Hospital of South China University

Song-Qing Wei

Changsha Central Hospital of South China University

Yong Yang

Changsha Central Hospital of South China University

Xiu-Ying Yi

Zhuzhou Central Hospital

Xin-Ping Jiang

Loudi Central Hospital

Hai-Ying Han

Shaoyang Central Hospital

Zhe-Feng Zhong

Nanhua Hospital,of South China University

Xiao-Ying Cao

Chenzhou Second People's Hospital

Feng-Jun L

Changde Second People's Hospital

Ge Zhou

Yueyang First People's Hospital

Xiao-Hui Gong

Xiangtan Central Hospital

Wen-Ting Zha

Hunan Normal University

He-Bin Xie

Changsha Central Hospital of South China University

Tuan-Mei Wang

Changsha Hospital for Maternal and Child Health Care

Man-Zhi Wang ( 41841703@qq.com)

Changsha Central Hospital of South China University

\section{Research Article}

Keywords: COVID-19, Pediatric infection, Epidemiology, Symptoms, Laboratory characteristics

Posted Date: September 16th, 2020 
DOI: https://doi.org/10.21203/rs.3.rs-78282/v1

License: (9) (i) This work is licensed under a Creative Commons Attribution 4.0 International License. Read Full License 


\section{Abstract}

Objective To investigate the epidemiological characteristics, clinical features, treatment and short-term prognosis of SARS-CoV2 infection in children.

Methods A retrospective analysis was conducted in children with SARS-CoV-2 admitted to twelve hospitals in eight cities in Hunan province, China, from January 26, 2020 to June 30, 2020.

Results A total of 48 children were enrolled in this study. 11 cases (23\%) were asymptomatic, 15 cases (31\%) were mild, 20 cases (42\%) were moderate, and 2 cases $(4 \%)$ were severe. No children were critical requiring intensive care. The most common symptom was fever (42\%), cough (40\%), fatigue (17\%) and diarrhea (10\%). The total peripheral blood leukocytes count decreased in two case (4\%), Lymphocytopenia was present in 5 cases (10\%). There were abnormal chest CT changes in 22 children (46\%), including 15 (68\%) with patchy ground glass opacity. In addition to supportive treatment, 41 children (85\%) received antiviral therapy, 11 patients and (23\%) were treated with antibiotics, 2 children (4\%) were treated with methylprednisolone and IVIG. There was no death occurred.

Conclusions Most children with SARS CoV-2 infection in Hunan province were asymptomatic, mild or moderate. Severe cases are rare. Close family contact was the main route of infection. The younger the age, the less obvious symptoms for children might be. Epidemiological history, nucleic acid test and chest imaging were important tools for the diagnosis in children.

\section{Introduction}

The emergence and spread of a novel coronavirus (SARS CoV-2) that occurred in Wuhan, Hubei province of China in December 2019, has resulted in a global health concern, threatening thousands of lives. The disease caused by SARS-CoV-2 is officially called Corona Virus Disease (COVID-2019). The World Health Organization (WHO) has declared COVID-19 as a pandemic. It has high contagiousness and rapid expansion through human-to-human transmissions ${ }^{1}$. As of June $30^{\text {th }}, 2020$, COVID-19 has been reported from more than 200 countries, affecting over 10 million persons and resulting in more than 500000 deaths ${ }^{2}$. Clusters is an important epidemiological characteristic of this outbreak ${ }^{3}$. At the emerging stage of the COVID-19, the infection starts from person-to-person transmission in the community, almost exclusively in adults. The virus further spreads to the family to cause intrafamilial transmission, especially to the elderly and children, who are vulnerable to the infection ${ }^{4}$. Although current reports indicated that most pediatric cases compared to adults were mild ${ }^{5}$, the potential risk of death cannot be ignored. During the previous outbreaks of SARS and MERS, ARDS and deaths occurred in children ${ }^{6-8}$.

In China, a total of 83,534 laboratory-confirmed cases had been documented through of June $30^{\text {th }}, 2020^{9}$. However, there have been still relatively few pediatric COVID-19 cases. A review of 44,672 laboratory-confirmed cases from across China as of February $11^{\text {th }}$, 2020, only $416(0.9 \%)$ were less than 10 years of age and $549(1.2 \%)$ between 10 and 20 years of age ${ }^{4}$. The 72,314 cases reported by the Chinese Center for Disease Control and Prevention had less than $1 \%$ as children younger than 10 years of age ${ }^{10}$. Although there has been more and more research on COVID-2019 since the outbreak, little information regarding the epidemiological characteristics and clinical features is available for children.

Hunan province borders on Hubei province (Wuhan as the capital), the epicenter of COVID-19 in China. Therefore, cases in Hunan province would provide a good basis to learn how COVID-19 spreads outside Wuhan. Here, we retrospectively analyzed the epidemiological history, clinical manifestations, treatment and prognosis of children infected with SARS CoV-2 in 8 different cities of Hunan province, in order to provide a guidance for clinical prevention and treatment.

\section{Methods}

\section{Data sources}

This retrospective review was conducted in 12 participating hospitals from 8 cities in Hunan province of China. We included all children $<18$ years of age who were diagnosed with COVID-19 between January $26^{\text {th }}$ and June $30^{\text {th }}, 2020$. The cases with SARS-CoV- 
2 infection were laboratory-confirmed by a real-time reverse-transcriptase polymerase-chain-reaction (RT-PCR) assay of nasal and pharyngeal swab specimens in the laboratory of the Chinese Center for Disease Prevention and Control (CDC).

\section{Clinical assessment and disease severity grouping}

We followed the recommendations issued by Pediatric Society of Chinese Medical Association for the diagnosis, prevention and control of SARS-CoV-2 infection in children $\left(1^{\text {st }} \text { interim edition }\right)^{3}$. We collected data on age, gender, exposure history, comorbid conditions, clinical symptoms, laboratory results, chest radiological features and treatments. Patients were divided into 5 groups: (1) Asymptomatic: no symptoms and no pneumonia on imaging; (2) Mild: only mild clinical symptoms with no pneumonia on imaging; (3) Moderate (referred as ordinary/common in the above-mentioned pediatric society recommendations): common symptoms and signs with typical pneumonia on imaging, or no clinical symptoms and signs but pneumonia on imaging; (4) Severe: the above typical features are accompanied by dyspnea and hypoxemia (oxygen saturation $\leq 92 \%$ ); (5) Critical: patients rapidly progressing to ARDS or respiratory failure, with shock, multiple organ dysfunction (MODS) and/or death.

\section{Statistical Analysis}

Data statistics were processed by SPSS 19.0 statistical software. Differences between groups were compared using the Chi-square tests and Fisher's exact probability method. Normal distribution measurement data are described by mean and standard deviation $(\bar{X} \pm s) . P<0.05$ was considered statistically significant.

\section{Results}

\section{Epidemiological characteristics}

A total of 48 pediatric cases with COVID-2019 from eight cities (Changsha, Zhuzhou, Loudi, Shaoyang, Hengyang, Chenzhou, Changde, and Yueyang ) of Hunan province were enrolled in this study. The distribution of age and sex as well as disease severity are showed in Table 1. The mean age of the 48 pediatric patients was 8.274 .66 years, ranged from 7 months to 17 years. Among them, only two cases (4\%) were < 1 year of age, 11 cases (23\%) were 1-3 years of age, 18 children (38\%) were 4-10 years of age and 17 children (35\%) were 11-17 years of age. $52 \%$ of cases were males, and the proportion of male to female was almost 1:1. None of the patients had underlying disease, and all of them had a history of documented epidemiological exposure. 16 (33\%) children were imported cases. Seven of them lived in Wuhan for a long time, 8 children had been to Wuhan within the past two weeks, and one child was infected on a cruise ship during January $19^{\text {th }}$ to $24^{\text {th }}, 2020$ from the port Guangzhou, China. No children had a history of exposure to Wuhan Huanan Seafood Wholesale Market. The familial cluster was confirmed in $46(96 \%)$ children. Only $2(4 \%)$ children hadn't been exposed to any confirmed adult patients, but they both lived in Wuhan for a long time.

\section{Clinical features}

As shown in Table 1, 11 children (23\%) were diagnosed as asymptomatic infection, 15 children (31\%) were mild type, and 20 children were moderate type ( $42 \%$, including 3 cases of subclinical type). Only $2(4 \%)$ patients had severe disease, and no cases were critical. As shown in Table 2, the most common symptom was fever (20 cases, $42 \%) .13$ children had low-grade fever $\left(37.3-38^{\circ} \mathrm{C}\right)$, only 3 children got high fever $\left(>39^{\circ} \mathrm{C}\right)$, and $28(58 \%)$ children had normal body temperatures throughout the course of COVID-2019. Eight children (17\%) had fatigue, $3(6 \%)$ children had headache and $3(6 \%)$ children had nasal congestion and runny nose. Cough (40\%) was another common symptom, including 4 (8\%) children coughed with sputum. 6 children (13\%) experienced gastrointestinal symptoms, including diarrhea (5 cases) and vomiting (1 case), and one child presented with diarrhea as the only symptom in the course. One patient developed convulsions accompanied by high fever $\left(>39^{\circ} \mathrm{C}\right)$, which didn't recur after fever disappeared. All the children had no discomfort such as dyspnea, hemoptysis and altered mental status, and didn't require intensive care support and invasive mechanical ventilation. We don't have accurate and detailed information on the clinical signs of the children, due to the difficulty for the medical staff wearing protective clothing to carry out a detailed physical examination of the children, especially for the lung auscultation.

\section{Laboratory findings}

All patients underwent routine peripheral blood counts, coagulation, myocardial enzyme, liver and kidney function tests. Compared to adults, children rarely have low levels of leucocyte and lymphocytes. 43 (90\%) patients had normal range of leucocyte, $3(6 \%)$ 
cases had elevated levels and $2(4 \%)$ case had low level. Lymphopenia (lymphocyte count $<1.5 \times 10^{9}$ per liter) was present in 5 (10\%) patients while $43(90 \%)$ cases had normal lymphocyte counts. All patients in this study had normal platelets and hemoglobulin. 3 children $(6 \%)$ were found to have elevated C-reactive protein $(>10 \mathrm{mg} / \mathrm{l})$. Elevated levels of procalcitonin $(>0.5 \mathrm{ng} / \mathrm{ml})$ and D-dimer ( $\geq 0.5 \mathrm{mg} / \mathrm{L}$ ) were both detected in 2 severe children (4\%). All children had normal levels of alanine aminotransferase, aspartate aminotransferase, creatine kinase, serum creatinine, urea nitrogen and blood glucose in our study. Serum ferritin and immune globulin were measured in 11 and 7 children, respectively and all of which were normal.

\section{Radiographic findings}

Of the 48 patients, 22 patients (46\%) showed lesions on chest CT scan (Table 2). Among them, 12 (55\%) patients had one lobe involvement, $10(45 \%)$ patients had more than two lobes involved. The most common radiographic finding was ground glass opacity. $15(68 \%)$ patients had ground glass opacity, 5 (22\%) patients had consolidation, and 2 (10\%) patients had mixed shadowing of ground glass and consolidation. None of the patients had pleural effusion. Twenty patients repeated a chest CT examination after 37 days. Among them, 4 (20\%) patients had no changes, 11 (55\%) patients had disease resolution, and $5(25 \%)$ patients had disease progression. All the imaging lesions of the 20 patients disappeared after 2 weeks of medical treatments.

\section{Medical treatments}

41children (85\%) received antiviral therapy. Among them, 24 (59\%) children were treated with a single antiviral drug as follows: interferon (atomized or sprayed nasal cavity, 7-10 days) in 9 children, lopinavir-ritonavir in 12 children, arbidol (oral, 3 times/d, 7-14d) in 2 children, and ribavirin (oral, 2 times/d, 10d) in one child. The remaining children were treated with a combination of two or more antivirus drugs, including oseltamivir phosphate (oral, 2 times/d, 5d), interferon, lopinavir-ritonavir, arbidol, ribavirin and traditional Chinese medicine. 11 children (23\%) were treated with antibiotics, six of whom with azithromycin (oral, 10mg/kg/d, 3-5d) for mycoplasma infection. Two children (4\%) were treated with methylprednisolone (intravenous, $2 \mathrm{mg} / \mathrm{kg} / \mathrm{d}, 3 \mathrm{~d}$ ) and IVIG (intravenous, $1 \mathrm{~g} / \mathrm{kg} / \mathrm{d}, 2 \mathrm{~d}$ ) for persistent high fever and pulmonary disease progression. None of the children received mechanically ventilations.

\section{Discussion}

Hunan province is adjacent to Hubei province (Wuhan as the capital city), and as of 24:00 on June $30^{\text {th }}, 2020$, a total of 1,019 confirmed cases had been reported in Hunan province. The government authority has taken unprecedented and effective effort to reduce the risk of transmission. Early diagnosis, early isolation and early management all contributed to reducing transmission and mortality in Hunan.

Person-to-person transmission and Cluster are important epidemic features of this outbreak, and close family contact is the main way of SARS-CoV-2 infection in children. Of the 16 imported cases, 15 children had a clear history of exposure in Wuhan, one child had cruise trip exposure. Of the 48 cases in our study, 46 (96\%) children had been exposed to confirmed adult patients and the other two children, who had been living in Wuhan, were imported cases. Compared to adults, children had atypical symptoms or were asymptomatic. In our study, $23 \%$ children were asymptomatic, indicating that the absence of clinical symptoms cannot rule out the diagnosis of infection. That's probably the reason why there were so few reported cases of COVID-19 in children and some asymptomatic pediatric patients were easily missed. Therefore, a detailed investigation of history of exposure to SARS-CoV- 2 is particularly important for children. In our study, there was no significant difference in incidence of SARS-CoV- 2 infection between male and female. $96 \%$ children in our study were predominantly asymptomatic, mild or moderate cases, with only 2 children who had severe disease, a 2-year old and a 4-year old. The age of the first critical pediatric patient of COVID-19 reported in China was one year old ${ }^{11}$. Although the incidence of severe and critical illness of SARS-CoV-2 infection in children is low, it seemed that the younger the age, the higher the incidence of severe and critical cases might be. This may be related to the fact that organ functions are not fully developed and complications are likely to occur at a younger age.

Most children of SARS-CoV-2 infection in our study had mainly upper respiratory tract symptoms, such as fever, cough, fatigue, headache, nasal obstruction and runny nose. $46 \%$ children had progression to lower respiratory tract infection, which mainly occurred in 4-10 years group and 11-17 years group. 13\% children in our research had gastrointestinal symptoms as vomiting and diarrhea, mainly in less than 3 years group and 4-10 years group, it showed a statistically significant difference. Gastrointestinal symptoms can be the only clinical manifestation in children, as shown in one child in this study, who presented with diarrhea as the only symptom in the disease course. Therefore, attention should be paid to some children without significant respiratory symptoms.

Page 5/10 
SARS-CoV-2 is rarely reported to involve the nervous system. In this study, a 2-year old girl without any underlying disease developed convulsions in the early stages of SARS-CoV-2 infection, which was accompanied by high fever $\left(>39^{\circ} \mathrm{C}\right)$ and didn't recur after fever disappeared. Although the child developed a severe case with typical ground glass opacity in both lungs, she did not show any neurological abnormalities. Therefore, we considered it was a febrile convulsion and was unrelated to SARS-CoV-2 infection.

Lymphocytopenia was common after the onset of SARS-CoV-2 infection in adults, but it was rare in children. In the initial routine blood tests of this group, there were only 5 cases with lymphocytopenia, three cases in the 4-10 years group and two cases in the 1117 years group. Thus, for children, especially young children, lymphocytopenia lacks the sensitivity to an early diagnosis for the SARS-CoV-2 infection. On the whole, other laboratory findings in children with SARS-CoV-2 infection showed no significant specificity and sensitivity and could not provide strong evidence for the diagnosis but could be used as a reference indicator to exclude other diseases. CRP is one of the indicators to assess inflammation, and an elevated CRP was present in $60.7 \%$ of COVID-19 adult patients, especially in severe patients ${ }^{2}$. In our study, only 3 children (6\%) had elevated CRP, and two of the children were severe and had elevated procalcitonin. It is likely that patients with severe COVID-19 are more susceptible to secondary bacterial infection than patients with mild disease.

Our study showed some common CT imaging features in patients infected by SARS-CoV-2, including ground-glass opacity of bilateral lobular and subsegmental areas of consolidation, or mixture, without pleural effusion (white lung appearance). Although the abnormal manifestations of chest CT in some children were typical, they still need to be differentiated from other viral pneumonitis. Therefore, pediatricians should carefully verify the findings according to epidemiological history, clinical manifestations, viral nucleic acid detection and CT images, in order to improve the diagnostic accuracy in children.

At present, there is no specific drug for COVID-19. Based on "Diagnosis and prevention of 2019 novel coronavirus infection in children (trial version 1)", the use of lopinavir/ritonavir (LPV/RTV) or ribavirin and interferon were recommended. Due to the difference in treatment regimens, dosages and combinations of antiviral drugs used different hospitals in this study group, it was difficult to determine the efficacy of antiviral drugs. The main treatment is mainly symptomatic and supportive care. If a child has a combined secondary bacterial infection, appropriate antibiotics may be used. It is highly recommended that appropriate antimicrobial agents should be selected after a full microbiological investigation, and timely adjustment should be made according to the curative effect and drug sensitivity test results, avoiding blind or inappropriate use of antibiotics. In reference to adult treatment experience in Wuhan city, routine use of corticosteroids should be avoided, and only reserved for severe cases with persistent high fever, CRP $\geq 30$ $\mathrm{mg} / \mathrm{L}$, serum ferritin $\geq 1000 \mu \mathrm{g} / \mathrm{L}$ or diffuse lesions in both lungs ${ }^{3}$. The efficacy of IVIG for COVID-19 pneumonia is limited in adult patients. In this study, after IVIG treatment, the pulmonary lesions of the two severe cases were all resolved. Whether the effect of IVIG in children is greater than that in adults still needs more data to prove.

In this study, due to the limited number of cases and short study time, we did not collect all pediatric cases in Hunan province. We may not have a comprehensive understanding of the clinical characteristics and imaging manifestations of pediatric patients, so a future study with larger sample size from multiple centers is needed.

In conclusion, the epidemiological characteristics of 48 children with COVID-19 in Hunan province in this study were mainly clustered cases. Most children with SARS CoV-2 infection in Hunan province were asymptomatic, mild or moderate. Severe cases are rare. The younger the patients, the less obvious their symptoms may be. Epidemiological history, nucleic acid test and chest imaging were important tools for the diagnosis of COVID-19 in children.

\section{Declarations}

\section{Acknowledgment}

We thank Dr. Ding-You Li, professor of pediatrics at Children's Mercy Kansas City, USA, for critical reading and editing this manuscript.

\section{Declaration of competing interest}

The authors declare that they have no known competing financial interests or personal relationships that could have appeared to influence the work reported in this paper.

\section{Funding information}


This work was supported by the COVID-19 special project of Changsha Science and Technology Plan, Grant/Award Number: kq2001011.

\section{Ethics Statement}

This study was performed in accordance with national ethics regulations and approved by the Medical Ethics Committee of our hospital (Batch No: 202003). The requirement for written informed consent of this retrospective study was waived by our institutional review board, since our data were de-identified and involved no potential risk to patients and no link between the patients and the researchers.

\section{Author contributions}

All authors have approved submission of the manuscript.

\section{References}

1. Guan, Z. Ni, Yu Hu, et al. Clinical Characteristics of Coronavirus Disease 2019 in China.N Engl J Med. 2020. PMID: 32109013

2. World Health Organization. Coronavirus disease (COVID-2019) situation reports. https://www.who.int/docs/defaultsource/coronaviruse/20200630-covid-19-sitrep-162. pdf?sfvrsn=e00a5466_2

3. Fang F, Zhao DC, Cheng Y, Luo XP. Recommendations for the diagnosis, prevention and control of the 2019 novel coronavirus infection in children (first interim edition). Chinese Journal of Pediatrics, DOI:10.3760/cma.j.issn. 0578-1310.2020.03.001. [Article in Chinese]

4. Cao Q, Chen YC, Chen CL, et al. SARS-CoV-2 infection in children: Transmission dynamics and clinical characteristics. J Formos Med Assoc. 2020 Mar; 119(3): 670-673. Doi: 10.1016/j.jfma.2020.02.009. Epub 2020 Mar 2.

5. Hong H, Wang Y, Chung HT, Chen CJ. Clinical Characteristics of Novel Coronavirus Disease 2019 (COVID-19) in Newborns, Infants and Children. Pediatr Neonatol. 2020 Mar 10. PMID: 32199864. [Online ahead of print]

6. Li ZZ, Shen KL, Wei XM, et al. Clinical analysis of pediatric SARS cases in Beijing. Chinese Journal of Pediatrics, 2003, 41(8):574577[Article in Chinese].

7. Zeng QY, Liu L, Zeng HS, et al. Clinical characteristics and prognosis of 33 children with severe acute respiratory syndrome in Guangzhou area. Chinese Journal of Pediatrics, 2003, 41(6): 408-412[Article in Chinese].

8. Thabet F, Chehab M, Bafaqih H, et al. Middle East respiratory syndrome coronavirus in children[J]. Saudi Med J, 2015, 36(4): 484486.

9. Covid-19 outbreak update as of 24:00 on 30 June 2020 from Chinese Center for Disease Prevention and Control (CDC). http://chinacdc.cn/jkzt/crb/zl/szkb_118 03/jszl_11809/202007/t20200701_217573.html.

10. Lu XX, Zhang LQ, Du H, et al. SARS-CoV-2 Infection in Children. N Engl J Med. 2020 Mar 18[Online ahead of print].DOI: 1056/NEJMc2005073.

11. Chen F, Liu ZS, Zhang Frist case of severe childhood novel coronavirus pneumonia in China[J/OL]. Chinese Journal of Pediatrics, 2020,58(2020-02-11). DOI: 10.3760/cma.j.issn.0578-1310.2020.0005.

\section{Tables}

Table 1 Demographic characteristics and disease severity distribution of 48 children infected with COVID-2019 


\begin{tabular}{|c|c|c|c|c|c|c|c|c|}
\hline & Asymptomatic & Mild & Moderate & Severe & Critical & Total (\%) & $x^{2}$ & $P$ \\
\hline \multicolumn{9}{|l|}{ Sex (\%) } \\
\hline Male & $5 \otimes 10 \bigotimes$ & $9819 \rrbracket$ & $10 \otimes 21 \rrbracket$ & $1 \otimes 2 \bigotimes$ & 0 & $25 \rrbracket 52 \rrbracket$ & $0.907 \#$ & 0.900 \\
\hline Female & $6 \otimes 13 \rrbracket$ & $6 \rrbracket 13 \rrbracket$ & $10 \otimes 20 \rrbracket$ & $1 \otimes 2 \bigotimes$ & 0 & $23 \rrbracket 48 \rrbracket$ & & \\
\hline \multicolumn{9}{|c|}{ Age group (\%) } \\
\hline$<1$ year & $1 \otimes 2 \rrbracket$ & 0 & $1 \otimes 2 \bigotimes$ & 0 & 0 & $2 \varangle 4 \rrbracket$ & 11.779\# & 0.152 \\
\hline 1-3years & $4 \llbracket 8 \rrbracket$ & $1 \otimes 2 \bigotimes$ & $5810 \bigotimes$ & $1 \otimes 2 \bigotimes$ & 0 & $11 \otimes 23 \rrbracket$ & & \\
\hline $4-10$ years & $1 \otimes 2 \rrbracket$ & $9819 \rrbracket$ & $7 \rrbracket 15 \rrbracket$ & $1 \otimes 2 \bigotimes$ & 0 & 18ه38ه & & \\
\hline 11-17 years & $5 \otimes 10 \bigotimes$ & $5810 \bigotimes$ & $7 \rrbracket 15 \rrbracket$ & 0 & 0 & $17 \otimes 358$ & & \\
\hline Total & $11 \otimes 23 \rrbracket$ & $15 \llbracket 31 \rrbracket$ & $20 \rrbracket 42 \rrbracket$ & $2 \bigotimes 4 \rrbracket$ & 0 & 48囚100ه & & \\
\hline
\end{tabular}

Note: \# used Fisher exact probability method

Table 2 Clinical Features and Radiographic Findings of 48 Children with COVID-2019 


\begin{tabular}{|c|c|c|c|c|c|c|}
\hline & $\leq 3$ years & $4-10$ years & $11-17$ years & Total (\%) & $x^{2}$ & $P$ \\
\hline \multicolumn{7}{|l|}{ Symptoms } \\
\hline Fever & 3 & 12 & 5 & $20(42)$ & 7.527 & $0.023^{*}$ \\
\hline $37.3-38^{\circ} \mathrm{C}$ & 1 & 5 & 7 & $13(27)$ & $5.675 \#$ & 0.174 \\
\hline $38.1-39^{\circ} \mathrm{C}$ & 1 & 2 & 1 & $4(8)$ & & \\
\hline$>39^{\circ} \mathrm{C}$ & 2 & 1 & 0 & $3(6)$ & & \\
\hline Fatigue & 0 & 3 & 5 & $8(17)$ & $4.483 \#$ & 0.101 \\
\hline Headache & 0 & 1 & 2 & $3(6)$ & 1.492\# & 0.620 \\
\hline Nasal congestion/Rhinorrhea & 1 & 1 & 1 & $3(6)$ & $0.521 \#$ & 1.000 \\
\hline Cough & 5 & 8 & 6 & $19(40)$ & 0.315 & 0.931 \\
\hline Sputum production & 0 & 1 & 3 & $4(8)$ & $2.583 \#$ & 0.362 \\
\hline Vomiting & 1 & 0 & 0 & $1(2)$ & $2.304 \#$ & 0.271 \\
\hline Diarrhea & 4 & 1 & 0 & $5(10)$ & $6.432 \#$ & $0.024 *$ \\
\hline Convulsions & 1 & 0 & 0 & $1(2)$ & $2.304 \#$ & 0.271 \\
\hline \multicolumn{7}{|l|}{ Abnormalities on chest CT scan $(n=22)$} \\
\hline Ground glass opacity & 5 & 5 & 5 & $15(68)$ & $2.594 \#$ & 0.710 \\
\hline Consolidation & 1 & 3 & 1 & $5(23)$ & & \\
\hline Mixed shadowing & 1 & 0 & 1 & $2(11)$ & & \\
\hline Lesions on one lobe & 2 & 7 & 3 & $12(55)$ & 5.634 & 0.061 \\
\hline Lesions on two and more lobes & 5 & 1 & 4 & $10(45)$ & & \\
\hline \multicolumn{7}{|l|}{ Laboratory tests } \\
\hline \multicolumn{7}{|l|}{ White blood cell count } \\
\hline $4.0-10.0 \times 10^{9} / \mathrm{L}$ & 10 & 18 & 15 & $43(90)$ & & \\
\hline$<4.0 \times 10^{9} / \mathrm{L}$ & 0 & 0 & 2 & $2(4)$ & & \\
\hline$>10.0 \times 10^{9} / \mathrm{L}$ & 3 & 0 & 0 & $3(6)$ & & \\
\hline \multicolumn{7}{|l|}{ Lymphocyte count } \\
\hline$<1.5 \times 10^{9} / \mathrm{L}$ & 0 & 3 & 2 & $5(10)$ & & \\
\hline$>3.2 \times 10^{9} / \mathrm{L}$ & 6 & 2 & 2 & $10(21)$ & & \\
\hline Blood platelet $<100 \times 10^{9} / \mathrm{L}$ & 0 & 0 & 0 & 0 & & \\
\hline Albumin $<40 \mathrm{~g} / \mathrm{L}$ & 0 & 0 & 3 & $3(6)$ & & \\
\hline Alanine aminotransferase $(\mathrm{ALT})>40 \mathrm{U} / \mathrm{L}$ & 0 & 0 & 0 & 0 & & \\
\hline Aspartate aminotransferase(AST) $>40 \mathrm{U} / \mathrm{L}$ & 0 & 0 & 0 & 0 & & \\
\hline Serum creatinine $>73 \mu \mathrm{mol} / \mathrm{L}$ & 0 & 0 & 0 & 0 & & \\
\hline Urea nitrogen $>7.14 \mathrm{mmol} / \mathrm{L}$ & 0 & 0 & 0 & 0 & & \\
\hline Creatine kinase $\geq 200 \mathrm{U} / \mathrm{L}$ & 0 & 0 & 0 & 0 & & \\
\hline D-dimer $\geq 0.5 \mathrm{mg} / \mathrm{L}$ & 2 & 0 & 0 & $2(4)$ & & \\
\hline
\end{tabular}




\begin{tabular}{|lllll|}
\hline Lactate dehydrogenase $(\mathrm{LDH}) \geq 250 \mathrm{U} / \mathrm{L}$ & 1 & 0 & 2 & $3(6)$ \\
\hline Blood glucose $>7.0 \mathrm{mmol} / \mathrm{L}$ & 0 & 0 & 0 & 0 \\
\hline C-reactive protein $\geq 10 \mathrm{mg} / \mathrm{L}$ & 1 & 2 & 0 & $3(6)$ \\
\hline Procalcitonin $\geq 0.5 \mathrm{ng} / \mathrm{ml}$ & 1 & 1 & 0 & $2(4)$ \\
\hline Serum iron protein $>400 \mathrm{ng} / \mathrm{ml}$ & 0 & 0 & 0 & 0 \\
\hline
\end{tabular}

Note: \#used Fisher exact probability method, * $P<0.05$ 